\title{
Review
}

\section{Application of CT Perfusion Imaging Technology in the Diagnosis of Hepatitis and Liver Cirrhosis}

\author{
Bo Li, Xiaodong Kang \\ Medical Imaging College, Tianjin Medical University, Tianjin China
}

\section{Keywords}

hepatitis, liver cirrhosis, hemodynamics,

tomography, X-ray computer, perfusion

\section{Correspondence}

Kang Xiaodong,

E-mail: kxd2004@125.com

DOI: $10.1515 / i i-2017-0140$

\begin{abstract}
Images obtained via computer tomography perfusion (CTP) technology, a non-invasive functional imaging method, can reflect the hemodynamic status and function of the liver. Images obtained via CTP imaging technology can be quantitatively analyzed. The fundamentals, examination, and analysis of CTP images are reviewed in this paper. In addition, this paper provides a review of normal liver CTP imaging, CTP research status, and future developments in the CTP imaging of hepatitis and liver cirrhosis.
\end{abstract}

Hepatitis and cirrhosis are common chronic liver diseases. Hepatitis gradually develops into liver cirrhosis. Lesions are reversible during the early pathogenic stage of liver cirrhosis but become difficult to reverse during later stages. Thus, accurate diagnosis and intervention are particularly crucial during the early stages of lesion development. Given the lack of obvious morphological changes associated with early-stage hepatitis, liver fibrosis, and liver cirrhosis, traditional imagebased diagnostic methods do not always accurately reflect liver anatomy. A previous study has shown that the liver undergoes hemodynamic changes during the early stages of hepatitis and liver cirrhosis ${ }^{[1]}$. These hemodynamic changes in the liver can be reflected and quantified via computer tomography perfusion (CTP) imaging technology for the early diagnosis of liver lesions. This paper provides a review of the fundamentals of CTP imaging and image processing. In addition, the CTP imaging of the normal liver, as well as the status of research on the clinical application and developmental prospects of CTP imaging in hepatitis and liver cirrhosis, is reviewed in this paper.

\section{Fundamentals, Examination, and Analysis of CTP Images}

CTP was first proposed by Miles et al. ${ }^{[2]}$ in 1991. In CTP, the time-density curve (TDC) of each pixel in a slice is acquired via the multiple intermittent, continuous CT scanning of the region of interest (ROI) of the selected slice after the intravenous bolus injection of a contrast agent. To evaluate the perfusion of tissues and organs, perfusion parameters, which are based on the different mathematical curve models, are used in image reconstruction and pseudocolor processing with the above parameters. The timedensity curve, which is the basis of CTP, refers to the curve acquired by perfusion dynamic scanning with the simultaneous intravenous injection of the contrast agent. The time-density curve describes the increasing density of each pixel in the slice with time. The abscissa represents time, and the vertical coordinate represents the $\mathrm{CT}$ value of the tissue after drug injection. The changes can indirectly reflect the blood perfusion volume of tissues and organs, as well as microvessel density, to provide a reference for the characteristics and position of the lesion. CTP was developed based on the principle of the dynamics of a nuclear medicine tracer to reflect the hemodynamic status and function of organs and tissues. CTP imaging is a non-invasive functional imaging technique that can be used to quantitatively assess tissue perfusion based on the image slice of a capillary, and to distinguish between components of the hepatic artery and portal vein.

The reported scanning procedures and programs for 
CTP are non-standardized because of the maturity and the different manufacturers of the technology. In general, however, for the first routine CT scan of the liver, a patient lies on their back and the fixed slices that require analysis are then selected. The fixed slices generally include liver lesions ${ }^{[3}$

${ }^{-6]}$. Representative slices are selected in the absence of clearly visible lesions. The commonly selected representative slice is the porta hepatis slice, which generally includes the liver, spleen, aorta, and portal vein. The mode of single-slice CT scanning is selected as the continuous dynamic scanning of the same slice, and the MSCT scanning mode is selected for multi-slice continuous dynamic scanning. Miles et al. ${ }^{[2]}$ scanned each slice 10 times for $7,10,13,16,21,31,37.5$, and $44 \mathrm{~s}$. The general number of scan times is $35-40$, and the scanning intervals vary with the amount of the contrast agent $\geq 40 \mathrm{ml}$. The contrast age is generally injected at a rate of $3-5 \mathrm{ml} / \mathrm{s}$. HE et al. ${ }^{[7]}$ used MSCT scan and injected the contrast agent at a rate of $0.5 \mathrm{ml} / \mathrm{kg}$. The scan was delayed for $8 \mathrm{~s}$ with an interval of $1 \mathrm{~s}$ and lasted for $50 \mathrm{~s}$. Weidekamm et al. ${ }^{[8]}$ performed CTP imaging with the contrast dose of 40 $\mathrm{ml}$ injected at the rate of $10 \mathrm{ml} / \mathrm{s}$. The injection and scanning were performed simultaneously. In the study, the researchers scanned once for every $2 \mathrm{~s}$ for a total of 16 times. They then scanned once for every $17 \mathrm{~s}$ for a total of 8 times. The entire process lasted for $88 \mathrm{~s}$.

With the progress of science and technology, CT machines are now equipped with image-processing software. If the imaging equipment does not include image-processing software, images can be quantified by using several calculation formulae, including non-deconvolution and deconvolution models. However, liver perfusion parameters obtained via different mathematical models are not the same. After the original data of the perfusion scan are entered into the image-processing software, the ROI will be drawn on the abdominal aorta, portal vein, and spleen based on software prompts. The software then automatically generates the relative TDC; form the corresponding perfusion pseudocolor images based on perfusion parameters; and analyze the selected ROI from pseudo-color images or softwaregenerated MIP images.

\section{Normal Liver CTP Imaging}

Different organs and tissues possess different hemodynamic characteristics and perfusion performances, thus allowing CTP images to reflect the hemodynamic status and function of organs and tissues. The liver is the most challenging organ to image via CTP because breathing movements disturb the liver during perfusion scanning. Moreover, the liver has a unique double blood supply system with multi-channel communication between the two systems. The hepatic artery system provides $20 \%-30 \%$ of the blood to the liver, whereas the remaining $70 \%-80 \%$ is provided by the portal vein system. During CTP imaging, the liver CT value first increases because of the entry of the contrast agent from the hepatic artery, and then increases because of the entry of the contrast agent from the portal vein. The components of the hepatic artery and portal vein can be objectively evaluated based on the difference in liver perfusion between the two pathways. Shintaku et al. ${ }^{[9]}$ examined 25 subjects, $88 \%$ in whom could the hepatic artery and portal vein blood flow could be clearly distinguished. Their results confirmed the feasibility of assessing liver diseases based on the hemodynamic changes of the hepatic artery and portal vein.

At present, the mathematical model of liver perfusion analysis mainly includes the non-deconvolution and deconvolution models. The non-deconvolution method is the main modeling method used with following main evaluation indexes: (1) Arterial liver perfusion (ALP); hepatic arterial perfusion (HAP): hepatic artery blood flow per unit of time and per $\mathrm{ml}$ of tissue ${ }^{[10]}$. (2) Portal venous perfusion (PVP); hepatic portal perfusion (HPP); and portal vein blood flow per unit of time and per milliliter of tissue $^{[11]}$. (3) Hepatic perfusion index (HPI): also known as hepatic artery fraction (HAF), that is, the volume of hepatic artery perfusion that accounts for a proportion of the total liver perfusion. (4) Hepatic portal perfusion index (PPI): portal vein perfusion volume accounted for the proportion of the total liver perfusion. (5) Total liver perfusion (TLP): the sum of hepatic artery perfusion and portal vein perfusion volume. (6) Blood flow: blood volume that flows through a certain amount of tissue per unit time. (7) Blood volume: the number of blood flows in the local area. This parameter is influenced by the size of the blood vessels and the number of open blood capillaries.

Since CTP imaging was first proposed, many scholars have investigated the optimal perfusion parameters for imaging the normal liver ${ }^{[12-17]}$. Researchers have reported that the following optimal values: HAP value of $0.17-0.358 \mathrm{ml} \cdot \mathrm{min}^{-}$ ${ }^{1} \cdot \mathrm{ml}^{-1}$; PVP value of $0.34-1.46 \mathrm{ml} \cdot \mathrm{min}^{-1} \cdot \mathrm{ml}^{-1}$; PPI value of 0.44-0.88; and HPI value of 0.04-0.33. After the CTP data 
are obtained, the corresponding pseudo-color images are constructed through the color scale assignment. Intensely red HAP, PVP, and HPI pseudo-color images indicate high perfusion volumes. The redder the TTP expression, the longer the time required to reach the peak. The blood flow state of tissues and organs is evaluated based on TPP. The TDC of each pixel in the slice reflects the changes in the concentration of the contrast agent in the organ. These changes indirectly reflect the changes in the volume of tissue organ perfusion.

\section{Research Status and Prospects of CTP for the Diagnosis of Hepatitis and Liver Cirrhosis}

Guan Jingsheng et al. ${ }^{[18]}$ utilized an experimental animal model to investigate the CTP imaging of hepatitis and early liver fibrosis. They reported statistically significant differences among the HAF, mean transit time (MTT), blood flow, and blood volume of the experimental groups. However, the period of hepatitis and liver fibrosis were not significantly different between groups. Furthermore, they reported that HAF and MTT increased significantly during the development of hepatitis to the early stage of liver cirrhosis. By studying normal rats and cirrhotic rats, Ma et al. ${ }^{[19]}$ found that the PVP and MTT of rats with liver cirrhosis rats decreased, whereas ALP increased.

Many scholars have used CTP imaging to identify the changes in the blood flow parameters of a disease-free liver, liver fibrosis, liver cirrhosis, and other diseases, and analyzed the correlation between changes in blood flow and disease severity. Jiang Li et al. ${ }^{[20]}$ conducted single-slice CTP imaging with 27 cases of liver cirrhosis patients and 13 control cases. The hepatic artery perfusion volume of Child A- and B-level patients decreased, whereas that of Child C-level patients increased. In addition, the hepatic artery perfusion index of Child C-level patients increased. The portal vein perfusion volume and total hepatic blood flow of Child B- and C-level patients decreased. Ronot et al. ${ }^{[21]}$ compared and analyzed CTP and pathological grading in 52 patients with hepatitis C. The PVP and THP of patients in the early and middle stage decreased, whereas MTT increased. These results indicated that CTP images could be used to differentiate between the early and middle stages of liver fibrosis. However, at present, the overlap between measurement data limits the clinical application of CTP. Using a 16-slice spiral CT liver perfusion scan, Long Liling et al. ${ }^{[22]}$ treated 31 cases of mild hepatic fibrosis (liver fibrosis stages S1 and S2), 34 cases of severe hepatic fibrosis (liver fibrosis stages S3 and S4) and/or early liver cirrhosis, and 42 cases of advanced liver cirrhosis with typical clinical symptoms and imaging performances, as well as a control group of 30 cases. A PVP value of $84.76-100 \mathrm{ml}$ $\cdot \mathrm{min}^{-1} \cdot \mathrm{ml}^{-1}$ was set as the diagnostic threshold for severe hepatic fibrosis and/or the early diagnosis of liver cirrhosis. They hold that CTP can facilitate the identification of severe liver fibrosis and/or early liver cirrhosis, as well as advanced liver cirrhosis.

Previous CTP scans were performed using multi-slice spiral CT with a single slice or 16 rows and below. Given the technical limitations of its narrow $\mathrm{Z}$-axis coverage, multislice spiral CT can only scan a few slices of the selected lesions, thus reflecting the partial perfusion information of the lesion but not the comprehensive perfusion situation. Given the selection of reference blood vessels and organs in CTP, previous CTP scans required that the scanned slice must be located near the first hepatic portal area. This limited perfusion analysis to the area around the lesion and prevented the perfusion scan of lesions that are distant from the first hepatic portal. In addition, analysis was limited by selected ROI range: lesions in the upper and lower slices of the ROI are easily missed, thus providing results with high errors. The spatial, temporal, and density resolution of CT have been significantly improved with the improvement of multi-slice spiral CT technology and the further refinement of the corresponding perfusion software. CT scan time and scanning conditions have been greatly reduced, enabling fullliver perfusion scan. The entire liver tissue can be included in the scan, and ROI selection is no longer limited. Through image reconstruction, ROIs in the transverse, sagittal, and coronal positions can be selected. Thus, corresponding hemodynamic information in the three positions can be shown.

How are the functions of the left and right lobes of the liver related? Are there any differences in the perfusion of the atrophic right lobe, the left lateral lobe, and the caudate lobe of patients with liver cirrhosis? Does compensatory hypertrophy in left lateral lobe indicate abnormal liver function? What perfusion parameters indicate the initiation of abnormal liver function? These topics are not or are rarely reported.

Liver puncture biopsy is the gold standard for the diagnosis of liver fibrosis and cirrhosis. This method is the 
important basis for the clear diagnosis of fibrosis, as well as the measurement of the inflammatory activity and the degree of fibrosis. Liver puncture biopsy is also the basis for drug selection for treatment ${ }^{[23]}$. However, the invasive examination procedure causes considerable trauma to the patient. In addition, multiple puncture sites are not recommended. Given the non-uniform development of liver parenchymal lesions, a single puncture point cannot be used for qualitative examination or can cause puncture errors, resulting in the over- or under-estimation of the degree of liver fibrosis. Pseudo-color images obtained from full liver volume perfusion can clearly show liver fibrosis, the distribution of liver cirrhosis nodules, and the situation of blood flow, thus greatly improving the accuracy of liver puncture biopsy.

With the rapid development of multi-slice spiral CT and the gradual improvement of software, CTP imaging has provided increasingly accurate hemodynamic clinical information for the early and accurate evaluation of the changes in liver microcirculation. However, given the nonstandardization of the current software and hardware for liver CTP and limited experience in the clinical application of high-end CT machines, the application of CTP technology in hepatitis and liver cirrhosis requires further investigation.

\section{Declarations}

\section{Acknowledgements}

No.

\section{Competing interests}

The authors declare that they have no competing interest.

\section{Authors' contributions}

B Li made the literature analysis and wrote, discussed and revised the manuscript of this review. XD Kang critically analyzed and corrected the manuscript. All authors read and approved the final manuscript.

\section{References}

1 Orrego H, Blendis LM, Crossley IR, et al. Correlation of intrahepatic pressure with collagen in the Disse space and hepatomegaly in humans and in the rat.Gastroenterology, 1981, 80(3): 546-556.

2 Miles KA, Hayball M, Dixon AK. Colour perfusion imaging: a new application of computed tomogeaphy. Lancet, 1991, 337(8742) :
643-645.

3 Materne R, Van Beers BE, Smith AM, el a1. Noninvasive quantification of liver perfusion with dynamic computed tomography and adual-input one compartmental model. Clin Sci, 2000, 99(6): 517-525.

4 Tsushima Y, Blomley JK, Kusano S, et al. The portal component of hepatic perfusion measured by dynamic CT: an indicator of hepatic parenchymal damage. Dig Dis Sci, 1999, 44(8): 1632-1638.

5 Tsushima Y, Unno Y, Koizumi J, el a1. Measurment of human hepatic and splenic perfusion using dynamic computed tomography: a preliminary report. Comput Methods Programs Biomed, 1998, 57(1/2): $143-146$.

6 Leggett DA, Kelley BB, Bunce IH, et al. Colorectal cancer: diagnostic potential of CT measurements of hepatic perfusion and implications for contrast enhancement protocols. Radiology, 1997, 205(3): 716-720.

$7 \mathrm{He}$ W, He Q. Hepatic perfusion parameters in cirrhosis: dynamic CT measurements correlated with portal vein CT angiography. In the Radiological Society of North America: RSNA 2003 Scientific Papers, 2003, Q08-1267.

8 Weidakmm C, Cejna M, Kramer L, et al. Effects of TIPs on liver perfusion measured by dynamic CT. AJR Am J oentgenol, 2005, 184( 2) : 505-510.

9 Shintaku K, Nakashige A, Ono C, et al. Appearance of the hepatic artery in multidetector-row CT using a test bolus injection. Nippon Igaku Hoshasen Gakkai Zaashi, 2001, 61(3): 100-102.

10 Miles KA, Hayball M, Dixion AK. Functional images of hepatic perfusion obtained with dynamic CT. Radiology, 1993, 188(2): 405-411.

11 Blomley MJ, Coulden R, Dawson P, et al. Liver perfusion studied with ultrafast CT. J Comput Assist Tomogr, 1995, 19(3): 424-433.

12 Shi L, Tian J, Wang P, et al. Pilot study of hepatic perfusion with multislice spiral CT. Chinese Journal of Hepatology, 2003, 11(9): 522-525.

13 Wang X, Chen W, Wu D, et al. Whole-liver perfusion scans with 64-slice spiral CT in patients with liver cirrhosis. Journal of Sichuan University: Medical Science Edition, 2011, 42 (3): 382-386.

14 Nakashige A, Horigechi J, Tamura A, et al. Quantitative measurement of hepatic portal perfusion by multidetector row $\mathrm{CT}$ with compensation for respiratory misregistrafion. Br J Radiol, 2004, 77(921): 728-734.

15 Jiang L, Yang JY, Li ZP. Hepatic perfusion parameters in liver cirrhosis: clinical application of CT perfusion imaging. In: the Radiologicat Society of Noah America: RSNA 2003 Scientific Papers, 2003, Q08-1266.

16 Van Beers BE, Leconte I, Materne R, et al. Hepatic perfusion parameters in chronic liver disease: dynamic CT measurements correlated with disease severity. AJR Am J Roentgenol, 2001, 176(3) : 667-673.

17 Tsushima Y, BlomLey MJ, Yokoyama H, et al. Does the presence of distant and local malignancy alter parechymal perfusion in apparently disease-free areas of the liver?. Dig Dis Sci, 2001, 46(10): 2113-2119.

18 Guan S, Zhao W, Zhou k, et al. Experimental study of CT perfusion in 
hepatitis, hepatic fibrosis and early stage of cirrhosis. Chinese Journal of Radiology, 2005, 39 (8): 877-881.

19 Ma G, Bai R, Jiang H, et al. Assessment of hemodynamics in a rat model of liver cirrhosis with precancerous lesions using multislice spiral CT perfusion imaging. Biomed Res Int, 2013: 813174.

20 Jiang L, Yang J, Xie H, et al. CT perfusion Hemodynamics study on liver cirrhosis: clinical application of CT perfusion imaging. Chinese Journal of Radiology, 2004, 38 (10): 1081-1086.

21 Ronot M, Asselah T, Paradis V, et al. Liver fibrosis in chronic hepatitis C virus infection: differentiating minimal from intermediate fibrosis with perfusion CT. Radiology, 2010, 256(1): 135-142.

22 Long L, Huang Z, Ding K, et al. The value of multi-slice spiral CT liver perfusion imaging to evaluate the chronic hepatic fibrosis and cirrhosis. Chinese Journal of Radiology, 2012, 46 (4): 317-321.

23 Chinese Society of Hepatology Liver Fibrosis Chemical Group. Consensus on evaluation of diagnosis and efficiency of hepatic fibrosis. Chinese Journal of Hepatology, 2002, 10 (5): 327-328. 